\title{
NanoCrystalline Cellulose, an environmental friendly nanoparticle for pharmaceutical application - A quick study
}

\author{
Yee Kai Song ${ }^{1,2}$, Irene Mei Leng Chew ${ }^{3}$, Thomas Shean Yaw Choong ${ }^{1}$ and Khang Wei Tan ${ }^{2}$ \\ ${ }^{1}$ Faculty of Engineering, University Putra Malaysia, 43400 UPM, Serdang, Selangor Darul Ehsan, Malaysia \\ ${ }^{2}$ Faculty of Engineering, Technology and Built Environment, UCSI University, Cheras, 56000 Kuala Lumpur, Malaysia. \\ ${ }^{3}$ School of Engineering, Monash University Sunway Campus, 46150 Bandar Sunway, Selangor Darul Ehsan, Malaysia.
}

\begin{abstract}
Nanocrystalline cellulose (NCC) is probably the most abundant nano material can be found in nature. It offers not only substantial environmental advantage due to its intrinsic biocompatibility and biodegradability, but also outstanding aspect ratio, tension modulus, bioavailability and permeability. NCC can be isolated from low value biomass through a well-developed acid hydrolysis method, and the structure can be readily manipulated during extraction to obtain desirable size distribution, surface charge and degree of crystallinity. Erratic absorption of drug has been a major issue for years until the emergence of nanoparticle adopted as smart vehicle for drug delivery. However, due to inevitably severe toxicity and the side effect from ordinary nanoparticle, the exploration of green nanoparticles are catching escalating attention is now in urge. Microcrystalline cellulose (MCC) has long been an option, nevertheless, due to their relatively low surface charge compared to total volume, drug release in a control manner was hardly satisfied. It is suggested that the above limitation can be overcome by nanocrystalline cellulose. This article discusses and explores their potential in cosmetic, personal care and pharmaceutical application, and the recent development in term of preparation and characteristic.
\end{abstract}

\section{Introduction}

The forest product industry has been consistently searching to unlock potentiality of green innovation, in which stimulated by the fact that industry and consumers are demanding green concept resources. Mohanty et al. [1] highlighted that green composite which has high biodegradability, high biocompatibility, high resource availability, and environmental friendly are able to compete with existing products made from petroleumbased material.

Cellulose is an organic component exists in cell walls of plants. It is constructed from repeating $\beta$-Dglucopyranose units and having three hydroxyl groups in each anhydroglucose unit (AGU) [2]. Cellulose is present in amorphous state, but it is also exhibits crystalline region depending on the location of hydrogen bonding [3]. Existence of hydrogen bonding gives hydrophilic property to cellulose yet maintaining insolubility in majority of solvents. Cellulose has constantly being a research subject as a green material, by focusing on the high functionality of chemical properties and mechanical properties. Development of nanotechnology over the years is capable of extracting cellulose at nanoscale with minimal defects on cellulose hierarchical structure, producing a new template of cellulose based material for the next generation [4].

Nanocrystalline cellulose (NCC) is a highly potential green and renewable nanomaterial. Ruiz et al. [5] reported that NCC is typically elongated rod-shape with dimensions ranging from 10 to $1000 \mathrm{~nm}$ in length and 1 to $100 \mathrm{~nm}$ in diameter. NCC has appeared in many terms, for instance nanowhisker, nanocrystals, microcrystals, nanofibrils, or cellulose nanocrystals. Different methods had been discovered in preparation of NCC. Acid hydrolysis is the most commonly used method to isolate amorphous region from crystalline region in cellulose [6]. The pretreatment, alkaline treatment, bleaching and acid hydrolysis process to obtain NCC from Oil Palm Empty Fruit Bunch (EFB) was attempted and published in the previous work [7]. As it can be observed from Dynamic Light Scattering (DLS) analysis, NCC particles received from EFB are generally having size much below $1 \mu \mathrm{m}$, Figure 1. In comparison, Song Y.K., et al. [8] studied its surface morphology using Field Emission Scanning Electron Microscope (FESEM), and it seems the extracted cellulose particles were slight larger than [7], Figure 2.

However, usage of acid hydrolysis has negative impacts, for example deterioration of NCC, acid corrosiveness, thermal instability, and incompatible to environment friendly concept. Nevertheless, NCC has few supreme properties that gained increasing attentions in recent years, this includes high specific strength, high tension modulus, high aspect ratio, large surface-tovolume ratio, large surface charge, bioavailability, biocompatibility, and competitive cost $[6,9]$. Several 
studies on NCC showed promising industry applications in various optical devices [10], regenerative medicine [11], automotive interior applications [12], and controlled drug delivery [13]. In this paper, only pharmaceutical and personal care applications are focused on.

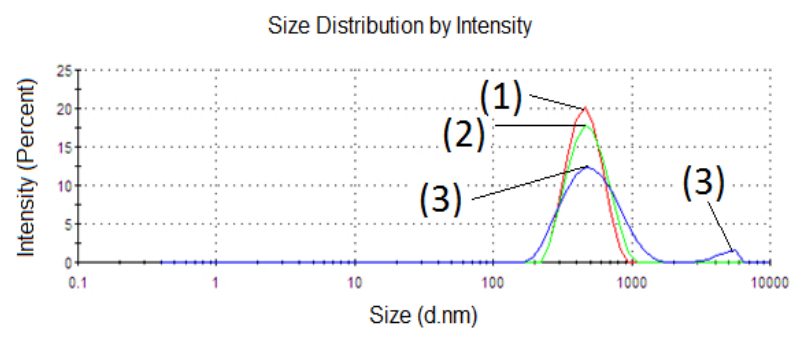

Figure 1. Particle size analysis using Malvern Zetasizer, Nano ZS Series [7]. It was conducted three time, i.e. measurement 1 , 2 and 3.

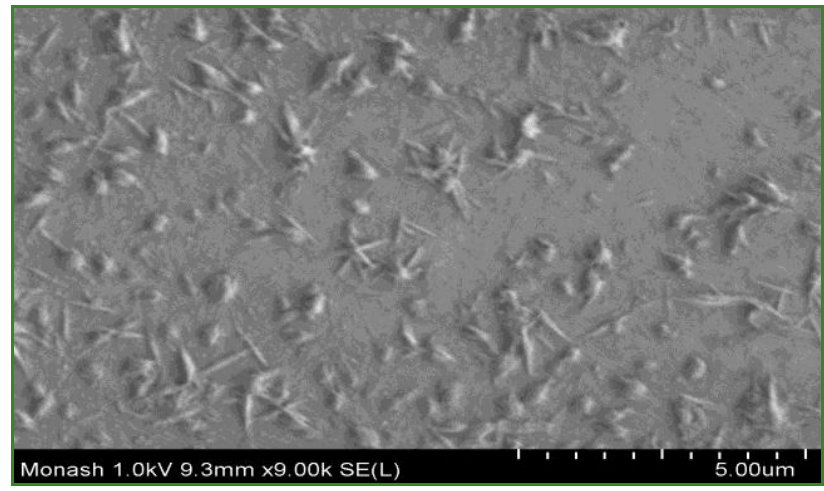

Figure 2. NCC extracted from bleached EFB fibers via ultrasonic cavitation [8]

\section{Potential application of NCC}

\subsection{Pharmaceutical}

Drug nanoparticles, or more commonly known as drug nanocrystals had been continuously a research topic in pharmaceutical application over the last two decades. Drug nanocrystals are often prepared in nanosuspension system consists of an aqueous medium and stabilizers. Majority of the pharmaceutical products are developed in the form of most stable solid crystalline. However, this gives rise to the problem of poor solubility, in which leading to low bioavailability [14]. The solution to this problem lies in the hydroxyl groups on the surface of NCC. The hydroxyl group can be altered via specific chemical modification such as surface charge modification, superior hydrophilicity, and enhance stability of matrix of NCC. The surface modification can be utilized to attract drugs to be attached on NCC.

\subsubsection{Drug disintergrant}

Microcrystalline cellulose (MCC) is widely used partially depolymerized cellulose as one of major component in pharmaceutical industry. MCC is blended with other excipients, for fabricating drug-loaded tablets for oral administration in the field of controlled drug delivery (CDD) technology. Excipient is inert substances that are functioned to initiate, propagate, and even take part in chemical interactions with active drugs compound. This role of excipients contributes directly to the effectiveness of pharmaceutical treatment. Gohel et al. [15] stated that MCC is not an excellent drug disintegrant due to its low porosity. Porosity of tablets affects significantly the degree of fluid penetration in aqueous environment, and disintegration of tablets [16]. Another drawback of using MCC in cellulose-based formulation is that it does not have high-accuracy control over the drug content release due to its poor surface charge density caused by relatively higher surface area of MCC. Hence, application of NCC instead of MCC is proposed to overcome the drawbacks mentioned. Jackson et al. [17] had performed an experiment that NCC is capable of binding significant quantities of ionizable water antibiotics, namely tetracycline and doxorubicin. Thus, usage of NCC as matrix for drug attachment is justified. Theoretically, NCC has higher porosity because surface area per total volume compared to MCC due to its smaller size. Even though the total surface area per unit total volume is higher in NCC, but the surface area per individual NCC is lower compared to $\mathrm{MCC}$, this directly contribute to higher surface charge density for superior molecularprecision control over drug content release.

\subsubsection{Fabrication of drug tablets}

Majority of oral drug administration in specific dosage is in the form of tablets. Tablets have been used widely in pharmaceutical due to its chemical and mechanical stability [18]. Fabrication of tablets has been a challenge in pharmaceutical industry as the requirements of a tablet is to have appropriate hardness, desired dissolution characteristic, and optimized drug content. Compression of functional ingredients with excipients is a conventional method of producing tablets. However, increase of pressure might cause higher friction generation between molecules, thus leading to higher temperature [19]. Consequently, thermal degradation occurred for thermalresponsive drug content and other active ingredients. Even though this problem can be overcome by usage of capsule to encapsulate thermal-responsive drugs instead of tablet, but this method is economically unfavorable compared to tablet [20]. Usage of NCC in tablet fabrication is a promising application to provide the desired requirements of tablet, at the same time overcome the negative effect of compression method to fabricate tablet. Robert et al. [21] reported that NCC has 7.5-7.7 GPa of tensile strength, $110-220 \mathrm{GPa}$ of elastic modulus in axial direction, and $10-50 \mathrm{GPa}$ of elastic modulus in transverse direction. Tensile strength of NCC is higher than Kevlar-49 fiber (3.5 GPa), carbon fiber (1.5 $5.5 \mathrm{GPa})$, and steel wire $(4.1 \mathrm{GPa})$. Elastic moduli in axial and transverse direction are also proven to be superior to Kevlar-49 fiber. These mechanical properties are suggested to have significant improvement on the 
hardness of tablets to consolidate the excessive frictions generated during compression.

\subsubsection{Fabrication of high tensile strength hydrogel}

Hydrogels have long been investigated for drug carrier as a biocompatible and swellable polymeric material [22]. The common applications of hydrogel are mostly explored in clinical usage, including tissue engineering, regenerative medicine, barrier materials to regulate biological adhesions, diagnostics, cellular immobilization, and separation of biomolecules or cells. The network structure possess by hydrogels can be non-porous, macroporous, microporous, and potential novel of nanoporous. Their highly porous structure can be manipulated by varying the density of cross-links in the gel matrix and the affinity of the hydrogels for the exposed aqueous environment in which they are subjected to swell. Hoare and Kohane [23] reviewed that the porosity of hydrogel also permits loading of drugs into the gel matrix and subsequent drug release at a rate dependent on the diffusion coefficient of the small molecule or macromolecule through the gel network. The reason why controllable drug release can be achieved in drug delivery is due to the cross-links in the gel matrix that are functioned in preventing the sudden burst of release of drug content. However, there is a major setback for controlled drug release application using hydrogel, which is low tensile strength. This setback does not just limit hydrogel usage use in load-significant applications, but also can resulted in extreme dissolution rate. This property does not favor in controlled drug delivery application. In recent drug delivery vehicle advancement, Zhang et al. [24] incorporated NCC into supramolecular hydrogel based on cyclodextrin inclusion to enhance mechanical properties and control drug release. The result showed a staggering increase in modulus as many as 5 times compared to the NCC-less hydrogel. The resulted nanocomposite, hydrogel containing NCC is found to be a potential drug carrier as mentioned previously.

\subsubsection{NCC matrix in transdermal drug delivery system}

Human skin is a readily route for drug delivery. The technology for non-oral drug delivery is known as transdermal drug delivery system (TDDS) which deliver appropriate amount of specific drug via the skin of a patient. The two biggest driving forces for development in drug delivery via skin are the convenience of patient and able to avoid first pass metabolism [25]. Other reported advantages of TDDS over conventional dosage form are controlled release of drug, more uniform plasma levels, improve bioavailability, control over drug administration duration using patches, and painless [2628]. The basic components for TDDS are [29]: (i) Polymer matrix/drug reservoir, (ii) Drug, (iii) Permeation enhancers, (iv) Pressure sensitive adhesive (PSA), (v) Backing laminates for patch protection, (vi) Release liner (vii). Other excipients such as plasticizers and solvents.
As mentioned earlier, NCC can be used as matrix former/drug reservoir, which is the main ingredient in formulation of TDDS. NCC matrix, in practical, is biocompatible and has potential compatibility via chemical surface modification with the drug, permeation enhancers, pressure sensitive adhesive (PSA), and other excipients. The outermost layer of skin epidermis is known as stratum corneum. It consists of 20 layers of lipids matrix in average to form a continuous structure. This continuous structure acts as skin barrier to avoid water loss. Each of stratum corneum cell is $40 \mu \mathrm{m}$ in diameter and $0.5 \mu \mathrm{m}$ thick [30]. First obstacle in TDDS is to penetrate stratum corneum layer, which require extremely small particle [31]. Matrix size contributes significantly to overall size of TDDS apart from drug molecular size. NCC is typically from 10 to $1000 \mathrm{~nm}$ in length and 1 to $100 \mathrm{~nm}$ in diameter, which means an approximate of 40 to 400 times smaller than the size of stratum corneum cell. Logically, usage of NCC should enhance the permeation percentage of TDDS across the skin. In addition, NCC matrix has several advantages over traditional polymer matrix, this include (i) Reduce efforts in formulating biocompatible polymers as drug reservoir. (ii) Avoid usage of health-risk synthetic polymer like polyvinylchloride and synthetic elastomers like rubber silicon. (iii) Smaller in size for ease of skin penetration. (iv) Higher precision in controlled drug release due to exceptional surface charge density.

\subsection{Why use NCC in pharmaceutical industry}

Potential of NCC in pharmaceutical applications has been an interesting research topic to replace micelles and liposomes in drug delivery system. Researches on NCC in drug delivery increase exponentially after few interesting toxicological assessment on NCC. One of the earliest toxicological assessments of NCC is conducted by Roman et al. [32] Human brain microvascular endothelial cells (HBMEC) was used as test substance. HBMEC are highly specialized cells responsible for the functional properties of the Blood-Brain Barrier (BBB). $\mathrm{BBB}$ is characterized by cellular tight junctions and active transport systems which allow the selective transport of substances into the brain while acted as a barrier to prevent unwanted substances from entering the brain. Hence, HBMEC is a good candidate to evaluate the toxicity on the drug uptake. NCC was found to be nontoxic to the cells. As for cellular uptake investigations, the results showed minimal uptake of untargeted NCC. Kovacs et al. [33] had done a very thorough toxicological assessment by using used two water fleas aquatic organisms, daphnia and ceriodaphnia, to compare the acute lethal toxicity of different nanomaterials. NCC and MCC are practically and virtually non-toxic. It is also found that no evidence of NCC interaction with other tissues, NCC was not passed down to next generation, and NCC is only restricted to digestive tract. Recently, Dong et al. [34] conducted a cytotoxicity assessment of nine different cell lines using 3-(4,5-dimethylthiazol-2yl)-2,5-diphenyltetrazolium bromide (MTT) assay and lactate dehydrogenase (LDH) assay. NCC showed no 
cytotoxic effects against any of these cell lines in the concentration range of $0-50 \mathrm{~g} / \mathrm{mL}$ and exposure time of 48 hours. Another investigation is conducted involving cellular uptake of fluorescein-5'-isothiocyanate (FITC)labeled NCC by these cell lines. The result showed that the cellular uptake is physiologically insignificant. These toxicological assessments concluded that lack of toxicity in NCC and untargeted NCC uptake strongly supports the potential of NCC in targeted drug delivery applications because it enables the application of specific uptake mechanism for drug targeting.

\section{Conclusion}

The applications and practices of NCC in pharmaceutical is still far beyond from its full potential. With the rapid advancement in mentioned industries, the exploration of green nanoparticles are catching escalating attention is now in urge. Nanocrystalline cellulose, or NCC is proposed to be an option, due to their relatively high surface charge density compared to total volume, high porosity, high tensile strength, high stability matrix former for various applications, and most importantly atoxic.

\section{Acknowledgement}

The authors are thankful to Ministry of Higher Education (MOHE) for the financial support through ERGS (ERGS/1/2013/TK07/UCSI/03/1), and CERVIE for RGS (PROJ-IN-FETBE-015).

\section{References}

1. Mohanty, A. K., Misra, M. \& Drzal, L. T. (2002). Sustainable bio-composites from renewable resources: Opportunities and challenges in the green materials world. Journal of Polymers and the Environment, 10(1/2), 19-26.

2. B. L. Peng, N. Dhar, H. L. Liu, \& K. C. Tam (2011). Chemistry and application of nanocrystalline cellulose and its derivative: A nanotechnology perspective. The Canadian Journal of Chemical Engineering, 89, 1191-1206.

3. Klemm D, Heublein B, Fink H-P, Bohn A. Angew Chem Int Ed 2005; 44, 2-37.

4. Robert J. Moon, Ashlie Martini, John Nairn, John Simonsen, \& Jeff Youngblood. (2011). Cellulose nanomaterial review: structure, properties, and nanocomposites. Chem. Soc. Rev., 40, 3941-3994

5. Ruiz M, M., Cavaillé, J. Y., Dufresne, A., Gérard, J. F., \& Graillat, C. (2000) Processing and characterization of new thermoset nanocomposites based on cellulose whiskers. Compos. Interfaces 7(2), 117-131.

6. Y. Habibi, L.A. Lucia, O.J. Rojas (2010) Cellulose nanocrystals: chemistry, selfassembly, and applications, Chem. Rev. 110, 3479-3500.

7. Shanmugarajah B., Kiew P. L., Chew M. L., Chong S. Y., Tan K.W. (2015) Isolation of nanocrystalline cellulose (NCC) from palm oil empty fruit bunch (EFB): Preliminary result on FTIR and DLS analysis. Chemical Engineering Transactions, 45, 1705-1710.

8. Song Y.K., Lim Y. H., Ho Y. L., Chek S.Y., Tan. K.W. (2013) NanoCrystalline Cellulose (NCC) as highly sustainable bio-engineered nanomaterial in solving energy, water and waste crisis at global scale. World Engineers Summits (WES), TUWAE, 9-15 Sept 2013.

9. M.M.D.S. Lima, R. Borsali (2004) Rodlike cellulose microcrystals: structure, properties and applications, Macromol. Rapid Commun. 25, 771-787.

10. Revol, J. F., Godbout, L., \& Gray, D. G. (1998). Solid self-assembled films of cellulose with chiral nematic order and optically variable properties. J. Pulp Pap. Sci. 24(5), 146-149.

11. K. Fleming, D.G. Gray, S. Matthews (2001) Cellulose crystallites, Chem. Eur. J. 7, 1831-1835.

12. Dahlke, B., Larbig, H., Scherzer, H. D., \& Poltrock, R. (1998). Natural fiber reinforced foams based on renewable resources for automotive interior applications. J. Cell. Plast. 34, 361-379.

13. Ruzica Kolakovic, Leena Peltonen, Antti Laukkanen, Jouni Hirvonen, Timo Laaksonen. (2012). Nanofibrillar cellulose films for controlled drug delivery. European Journal of Pharmaceutics and Biopharmaceutics 82, 308-315.

14. Lipinski C. Poor aqueous solubility - an industry wide problem drug discovery. Am Pharm Rev 2002, 5, 82-85.

15. Preparation and assessment of novel coprocessed superdisintegrant consisting of crospovidone and sodium starch glycolate: a technical note. AAPS PharmSciTech 8, Article 13.

16. Nogami, H., Nagai, T. and Uchida, H. (1966) Studies on Powdered Preparations XIV. Wetting of Powder Bed and Disintegration Time of Tablet. Chem. Pharm. Bull 14, 152-158.

17. Jackson J.K., Letchford. K., Wasserman B.Z., et al. (2011). The use nanocrystalline cellulose for the binding and controlled release of drugs. International Journal of Nanomedicine. 6, 321-330.

18. Joshi, X. Duriez (2004) Added functionality excipients: an answer to challenging formulations, Pharmaceutical Technology, 12-19.

19. H. Abushammala, R. Hashaikeh, C. Cooney (2012) Microcrystalline cellulose powder tableting via networked cellulose-based gel material. Powder Technology 217, 16-20.

20. C. Shaine, Pharmaceutical manufacturing handbook: Production \& process, 2008.

21. Robert J. M., Ashlie M.,John N.,John S.,Jeff Y. (2011) Cellulose nanomaterial review:structure, properties, and nanocomposites. Chem. Soc. Rev. 40, 3941-3994.

22. Amin, S., Rajabnezhad, S. and Kohli, Kanchan (2009) Hydrogels as potential drug delivery systems. Scientific Research and Essay, 3 (11), 1175-1183, 2009.

23. Haore, T. R. and Kohane, D. S. (2008) Hydrogels in drug delivery: Progress and challenges. Polymer 49, 1993-2007. 
24. Zhang, X. L., J. Huang, P. R. Chang, J. L. Li, Y. M. Chen, D. X. Wang, J. H. Yu and J. H. Chen (2010) Structure and Properties of Polysaccharide Nanocrystal-Doped Supramolecular Hydrogels Based on Cyclodextrin Inclusion," Polymer 51, 4398-4407.

25. Loyd V. Allen Jr, Nicholas G. Popovich, Howard C. Ansel (2005) Pharmaceutical dosage forms and drug delivery systems, 8th Edition., Wolter Kluwer Publishers, New Delhi, 298-299.

26. Robinson J. R., Lee H. L. Controlled drug delivery fundamentals and application. 2nd ed. Marcel Dekker, New York. 524 - 552.

27. Singh J., Tripathi K.P., Sakia T.R. (1993) Effect of penetration enhancers on the in vitro transport of ephedrine through rat skin and human epidermis from matrix based transdermal formulation. Drug Dev. Ind. Pharm, 19, 1623-1628.

28. Aquil M., Sultana Y., Ali A. (2003) Matrix type transdermal drug delivery systems of metoprolol tartrate: In vitro characterization. Acta Pharm, 53, $119-125$.

29. Nikhi S., Bharat P., Shalini S., Uday M. (2012) Blooming pharma industry with transdermal drug delivery system. Indo Global Journal of Pharmaceutical Sciences 2(3), 262-278.

30. A. W Kenneth, Dermatological and transdermal formulations, New York, USA: Marcel Dekker Inc; 2002

31. Debjit B., Chiranjib, Margret C., Jayakar B., Sampath K.P. (2010) Recent advances in transdermal drug delivery system. International Journal of PharmTech Research. 2, 68-77.

32. Roman M., S. P. Dong, H. Anjali, Y. W. Lee (2010) Cellulose Nanocrystal for drug delivery. Pollysaccharide Materials: Performance by design. Ametican Chemical Society, Washington DC, 81-91.

33. Kovacs T., V. Naish, B. O'Connor, C. Blaise, F. Gagne, L. Hall, V. Trudeau, P. Martel (2010) An ecotoxicological characterization of nanocrystalline cellulose (NCC). Nanotoxicol. 4(3), 255-270 (2010).

34. Dong S.P., Anjali A. H., Katelyn R. C., Lee Y. W., Roman M. (2012) Cytotoxicity and cellular uptake of cellulose nanocrystals. World Scientic Publishing Company. Nano LIFE, 2, No. 3. 